\title{
Cholesterol-lowering effects of barley dietary fibre in humans: scientific support for a generic health claim
}

\author{
Per Åman
}

Department of Food Science, Swedish University of Agricultural Sciences, Uppsala, Sweden

Abstract

New research has shown that foods containing whole-grain barley or certain dry-milled ingredients from barley have cholesterol-lowering effects similar to those seen for certain oat foods. The US Food and Drug Administration therefore allows whole-grain barley and certain dry-milled barley fractions to claim a reduction in the risk of coronary heart disease. A new Swedish study has been performed with a specific boiled, flaked and milled barley product, Aktivated Barley ${ }^{\circledR}$, mixed into cold drinks. This study showed a reduction in low-density lipoprotein-cholesterol levels in mildly hypercholesterolaemic subjects. There is therefore enough evidence for an extension of the generic health claim in the Swedish Code on health claims about certain types of dietary fibre, with regard to blood cholesterol levels and cardiovascular disease, to include barley fibres. The extension should also include foods with both oat and barley fibres, since there is no reason to believe that the mechanism(s) behind the cholesterol-lowering effect differ between the two cereal fibres.

Keywords: Barley; cholesterol; dietary fibre; $\beta$-glucan; health claim

$\mathrm{H}$ igh cholesterol levels in the blood represent an independent diet-related risk factor for atherosclerosis and are thereby connected with cardiovascular diseases. It is well known that certain types of dietary fibre, such as partly soluble $\beta$-glucan and psyllium fibre, help to reduce the cholesterol values.

\section{Previous evaluation by SNF}

A few years ago, the research committee of SNF (www.snf.ideon.se) evaluated the cholesterol-lowering effects of certain soluble dietary fibres in foods (1). The conclusion was that for oat fibres there is enough scientific evidence for a generic health claim to be used. Therefore, the Swedish Code on health claims (2) allows claims to be used according to the two-step principle for oat foods containing $0.75 \mathrm{~g}$ $\beta$-glucan per serving or $3 \mathrm{~g}$ per day at normal consumption. An example of such a claim is, "A nutritionally balanced diet high in soluble fibres from oats can contribute to lower cholesterol levels in the blood and thereby to a reduced risk of cardiovascular disease. This product is high in soluble oat fibres ( $\beta$-glucan)". For foods containing $\beta$-glucan that has been (or can be expected to be) degraded during processing (3), evidence for preserved cholesterol-lowering properties must be given. It was acknowledged that barley (mainly $\beta$ glucan) and rye (mainly arabinoxylan) were also rich in partly soluble fibres and that certain studies had shown cholesterol-lowering effects. The conclusion for food containing these cereals was, however, that more studies are necessary before generic claims can be approved (1).

\section{FDA approval of a generic claim for barley}

It is well known today that barley is rich in partly soluble dietary fibres, mainly $\beta$-glucan. In normal dehulled barley used for human consumption, the content of $\beta$-glucan generally varies between 3 and $7 \%$, which is within the same range as for oats, but barley cultivars with very high content $(14 \%)$ have also been identified (4). Recently, the US Food and Drug Administration (FDA) allowed barley products to claim a reduction in the risk of coronary heart disease as part of its continuing initiative to provide Americans with the information that they need to make healthy nutritional choices (5). The 
FDA concluded that risk factors for cardiovascular disease include high total cholesterol levels and high levels of low-density lipoprotein (LDL)-cholesterol, and that scientific evidence shows that adding whole-grain barley (dehulled or hull-less) or certain dry-milled barley products to food can contribute to lowering serum cholesterol. It was stated that the effect of barley foods was in the same range as for oat foods.

The FDA approval was based on a health claim petition submitted by the National Barley Food's Council, including 11 human clinical studies. Six of those were, however, excluded from the review by the FDA for various reasons. Consequently, the health claim is based on five human intervention studies supporting the relationship between a reduced risk of coronary heart disease and consumption of barley $\beta$-glucan soluble fibre (6-10). Three of the studies concerned hypercholesterolaemic men, one study concerned mildly hypercholesterolaemic men and women, and one women only. In the study by Behall et al. (7), 25 men and 25 women were given test diets providing 0,3 or $6 \mathrm{~g}$ per day of barley $\beta$-glucan, but with approximately the same amount of total dietary fibre. In comparison with the $0 \mathrm{~g}$ per day $\beta$-glucan diet period, there were statistically significant reductions in serum total cholesterol following both the 3 and $6 \mathrm{~g}$ per day $\beta$-glucan diets (5 and $6 \%$ reductions, respectively), as well as in serum LDL-cholesterol (10 and 13\% reduction, respectively). Serum highdensity lipoprotein (HDL)-cholesterol levels did not differ between the test periods. The other four studies were performed with between 10 and 21 men or women, and thus were all relatively small.

\section{Additional supportive studies}

The effects of barley $\beta$-glucan on cardiovascular and diabetic risk were reviewed recently (11). In this review an unpublished study with isolated barley $\beta$ glucan was briefly presented. Concentrated $\beta$-glucan extracts with high or low molecular weight (molecular weights are not given) added to food products were reported efficiently to lower LDLcholesterol in hyperlipidaemic subjects with and without the metabolic syndrome. After 6 weeks of treatment, mean LDL-cholesterol levels fell by $15 \%$ in the group that received $5 \mathrm{~g}$ high molecular weight $\beta$-glucan per day, $13 \%$ in the group that received $5 \mathrm{~g}$ low molecular weight $\beta$-glucan per day and $9 \%$ in both of the groups that received $3 \mathrm{~g}$ of $\beta$-glucan (high or low molecular weight $\beta$-glucan) per day. These results showed that $5 \mathrm{~g}$ of $\beta$-glucan per day was more effective than $3 \mathrm{~g}$ per day, and that in this study molecular weight did not have a major effect on the results. Inconsistencies in human data, however, led the authors to conclude that additional research is required for the clarification of certain questions.

Cholesterol-lowering effect of a new barley ingredient A new barley product (Aktivated Barley ${ }^{\circledR}$, NutriTech International AB, Kristianstad, Sweden) has been developed and part of the process has been patented (12). The process includes boiling, flaking and milling of barley kernels. The milled product is distributed in $20 \mathrm{~g}$ packages and contains $5.0 \%$ $(1.0 \mathrm{~g}) \beta$-glucan of high average molecular weight $\left(1.7 \times 10^{6} \mathrm{~g} \mathrm{~mol}^{-1}\right)$. The molecular weight distribution is similar to that of $\beta$-glucan in unprocessed barley kernels. The product ( $20 \mathrm{~g}$ on three different occasions per day) has been tested in a cross-over design, single-blind, randomized, placebo-controlled human study with 49 subjects, of whom 41 completed the study (13). The primary objective was to determine the effect on LDL-cholesterol level after 4 weeks of consumption in comparison to placebo, which was an instant flour with added cellulose. Both products contained about $11 \%$ total dietary fibre. The subjects were mildly hypercholesterolaemic women and men with LDL-cholesterol levels $>3.0 \mathrm{mmol}^{-1}$. Owing to laboratory failure, only 39 subjects were analysed for efficacy. Secondary objectives were to study the effect of Aktivated Barley on HDL and total cholesterol, triglycerides, C-reactive protein, blood glucose, insulin and safety.

The study showed a significant $5 \%$ reduction $(p=0.0031)$ in LDL-cholesterol level after 4 weeks consumption in comparison to placebo. Total cholesterol and triglyceride levels were also significantly reduced. The conclusion of the study was that the studied population was likely to achieve health benefits by reducing their LDL and total cholesterol levels when consuming the processed barley product. 


\section{Negative studies}

Cardiovascular disease risk factors in mildly hypercholesterolaemic men $(n=18)$ were studied in a randomized, controlled, cross-over study with enriched barley $\beta$-glucan (14). The preparation (Glucagel) used contained $75 \% \quad \beta$-glucan, which was insoluble in cold water and had a weak gelforming capacity. Active treatment comprised a daily supplement of $0.67 \mathrm{~g} \beta$-glucan $\mathrm{MJ}^{-1}$ in different foods. There was no statistically significant change in total cholesterol $(-1.3 \%)$ or LDLcholesterol $(-3.8 \%)$ when compared between treatments. During the preparation of Glucagel, the molecular weight of the polysaccharide is highly degraded by endogenous enzymes in the barley. The low

molecular

size could, at least partly, explain the lack of effect. In an unpublished experiment from the author's laboratory, a Glucagel preparation was found to have an average molecular weight of only about $100000 \mathrm{~g} \mathrm{~mol}^{-1}$.

In another study (15), barley was milled and treated with enzymes, and insoluble fibres were removed. Such preparations did not lower total or LDL-cholesterol levels when incorporated into drinks, which was probably also due to a low average molecular weight (40000 $\mathrm{g} \mathrm{mol}^{-1}$ ).

\section{Conclusions}

There is enough evidence for an extension of the claim about certain types of dietary fibre and cholesterol-lowering effects according to the Swedish Code to include barley fibres. Barley products subject to the claim should provide $0.75 \mathrm{~g}$ of $\beta$ glucan per serving or $3 \mathrm{~g}$ per day in normal consumption, as for oat products. The cholesterollowering effects of products that have been treated in wet processes should be verified. Since the active fibre component in both oat and barley foods is believed to be $\beta$-glucan, the claim can also be used for foods containing ingredients from both cereals.

When consumed in cold drinks Aktivated Barley ${ }^{\circledR}$ showed an expected effect on cholesterol levels in mildly hypercholesterolaemic subjects. The cholesterol-lowering effect after processing has therefore been verified for this specific ingredient, and a generic claim about barley fibres and cholesterol-lowering effect can be applied to dry mixtures providing $0.75 \mathrm{~g} \beta$-glucan per serving, or $3 \mathrm{~g}$ per day in normal consumption.

\section{References}

1. Asp N-G, Åman P. Vetenskaplig documentation av hälsopåståenden. Kolesterolsänkande effekt av lösliga fibrer. Scand J Nutr 2000;44:86-7. (In Swedish.)

2. Health Claims in the Labelling and Marketing of Food Products. The Food Sector's Code of Practice, 2004. Available at: www.hp-info.nu and www.snf. ideon.se

3. Åman P, Rimsten L, Andersson R. Molecular weight distribution of $\beta$-glucan in oat-based foods. Cereal Chemistry 2004; 81: 356-60.

4. Åman P, Graham H. Analysis of total and insoluble mixed-linked $(1 \rightarrow 3),(1 \rightarrow 4)-\beta$-D-glucans in barley and oats. J Agric Food Chem 1987; 35: 704-9.

5. Food and Drug Administration. http://www.fda.gov/ bbs/topics/NEWS/2006/NEW01375.html

6. Behall KM, Scholfield D, Hallfrisch J. Lipids significantly reduced by diets containing barley in moderately hypercholesterolemic men. J Am Coll Nutr 2004; 23: 55-62.

7. Behall KM, Scholfield DJ, Hallfrisch J. Diets containing barley significantly reduce lipids in mildly hypercholesterolemic men and women. Am J Clin Nutr 2004; 80: 1185-93.

8. McIntosh GH, Whyte J, McArthur R, Nestel PJ. Barley and wheat foods: influence on plasma cholesterol concentrations in hypercholesterolemic men. Am J Clin Nutr 1991; 53: 1205-9.

9. Newman RK, Newman CW, Graham H. The hypercholesterolemic function of barley beta-glucans. Cereal Foods World 1989; 34: 883-6.

10. Li J, Kaneko T, Qin L-Q, Wang J, Wang Y. Effects of barley intake on glucose tolerance, lipid metabolism, and bowel function in women. Nutrition 2003; 19: 926-9.

11. Pin JJ, Kaur H. A review of the effects of barley $\beta$-glucan on cardiovascular and diabetic risk. Cereal Foods World 2006; 51: 8-11.

12. Method of treating barley. International patent. Publication No. WO 02/074101 (A1). World Intellectual Property Organization; 26 September 2002. http://v3.espacenet.com

13. Clinical study report: Effects of a boiled, flaked, milled barley powder product (Aktivated Barley ${ }^{\circledR}$ ) on LDL-, HDL- and total cholesterol, triglycerides, glucose, insulin and HS-CRP levels in healthy hypercholesterolemic men and women. Uppsala: Centre for Human Studies of Foodstuffs (KPL). (Sponsored by NutriTech International AB, Kristianstad, Sweden.)

14. Keogh GF, Cooper GJS, Mulvey TB, McArdle BH, Coles GD, Monro JA, Poppitt SD. Randomized controlled crossover study of the effect of a highly $\beta$-glucanenriched barley on cardiovascular disease risk factors in 
mildly hypercholesterolemic men. Am J Clin Nutr 2003; 78: 711-8.

15. Biörklund $M$, van Rees A, Mensink RP, Önning G. Changes in serum lipids and postprandial glucose and insulin concentrations after consumption of beverages with $\beta$;-glucans from oats and barley: a randomised dose-controlled trial. Eur J Clin Nutr 2005; 59: 1272-81.
Per Åman

Department of Food Science

Swedish University of Agricultural Sciences

SE-750 07 Uppsala

Sweden

E-mail: per.aman@|mv.slu.se 